\title{
Maximum Torque-per-Amp Tracking Control of Saturated Induction Motors
}

\author{
Sergei Peresada, Serhii Kovbasa, Serhii Dymko \\ Department Electrical Engineering, National Technical University of \\ Ukraine "Igor Sikorsky Kyiv Polytechnic Institute" \\ Kyiv, Ukraine \\ sergei.peresada@gmail.com
}

\author{
Sergiy Bozhko \\ Faculty of Engineering, University of Nottingham, University Park \\ Nottingham NG7 2RD, United Kingdom
}

\begin{abstract}
An improved maximum torque per Ampere (MTPA) controller for induction motor (IM) drives is presented. The proposed MTPA field oriented controller guarantees asymptotic torque tracking of smooth reference trajectories and maximises the torque per Ampere ratio when the developed torque is constant or slow varying. Due to use closed loop flux observer and high-gain PI controllers for both stator current components the proposed solution provides improved robustness with respect to parameters variations and inverter non-idealities. Experimental tests prove the accuracy of the proposed control over a full torque range. In addition, a higher torque per Ampere ratio is achieved together with an improved efficiency of electromechanical energy conversion.
\end{abstract}

Keywords-induction motor, torque, control, tracking, saturation.

\section{INTRODUCTION}

Vector controlled induction motor (IM) drives typically operate with constant flux magnitude even at low values of produced torque, which results in good dynamic performance [1]. However conversely, the machine efficiency and power factor can be low, especially for small torque values.

The optimization techniques typically reported in publications adjust the flux level as a function of the electromagnetic torque using various optimization procedures. A number of control strategies to optimize different performance objectives in steady state are known [2], [3], including minimization of active and total losses, power factor maximization, MTPA control, maximum torque per voltage control and maximum power transfer. Under the MTPA control strategy, the torque controller adjusts the flux reference to minimize the stator current for a given machine torque and therefore increases the efficiency at low loads [4]. The basic MTPA control objective is achieved by controlling stator current torque and flux components, expressed in terms of rotor flux reference frame, to be equal.

For all the optimization techniques above an important issue for variable flux operation is the machine saturation effect. This effect results in varying machine inductances hence the assumption of linear magnetic circuits, common for standard optimization routines, is no longer valid. The nonlinear saturation effect is taken into account in [5], [6] for MPTA controller development using stored computed or measured data.

The asymptotic torque tracking control with MTPA optimization for saturated IMs has been proposed in [7] where nonlinear saturation effect is taken into account in both tracking controller design and flux reference computation. In contrast to standard direct field oriented controllers the authors proposed to use the closed loop flux observer on the base of flux current regulation error. Such structure provides some robustness properties with respect of the rotor circuit parameters variation if motor speed is not zero. However, for small speeds a degradation of the d-axis current loop performance is observed due to action only the proportional current feedback in the loop.

This study, in order to improve the torque-flux tracking performance and controller robustness, proposes to use a robust third order flux and d-axis stator current observer for saturated IM model. For such structure, the estimation and regulation processes are separated allowing to implement the both stator current controllers with robust PI structure. The key contribution of this paper is a novel torque-flux tracking controller design that simultaneously provides asymptotic torque tracking of the smooth reference trajectories in the whole range of machine torques and robust tracking of the torquedependent flux references in order to achieve MTPA optimization in steady state. The theoretical findings of this study and the effectiveness of the proposed approach are confirmed by thorough experimental validation.

\section{INDUCTION MACHINE MODEL}

For the purpose of this study the $1 / \lambda$-saturated IM of E. Levi (see [7]) has been employed. The model assumes that only the magnetizing inductance $L_{m}$ is saturated hence the leakage inductances are constant, and neglects the cross-saturation inductance so static and dynamic magnetizing inductances are equal.

The following definitions are used: $L_{m}\left(i_{m}\right)=\psi_{m}\left(i_{m}\right) / i_{m}$ is a static inductance of the magnetizing circuit, $L_{l}\left(i_{m}\right)=L_{m}\left(i_{m}\right)+L_{1 \sigma}$, $L_{2}\left(i_{m}\right)=L_{m}\left(i_{m}\right)+L_{2 \sigma}$ are the stator and rotor inductances, respectively, $\psi_{m}\left(i_{m}\right)$ - magnetizing curve, $i_{m}$ - magnetizing current, $L_{1 \sigma}=$ const and $L_{2 \sigma}=$ const are stator and rotor leakage inductances. Under these assumptions, the two-phase model of saturated IM in an arbitrary rotating reference frame, $(d-q)$, is given as follows:

$$
\begin{aligned}
& \dot{\omega}=\left(T-T_{L}\right) / J, T=\mu_{1 m}\left(\psi_{d} i_{q}-\psi_{q} i_{d}\right) ; \\
& \dot{i}_{d}=-\gamma_{m} i_{d}+\omega_{0} i_{q}+\alpha_{m} \beta_{m} \psi_{d}+\omega \beta_{m} \psi_{q}+u_{d} / \sigma_{m} ; \\
& \dot{i}_{q}=-\gamma_{m} i_{q}-\omega_{0} i_{d}+\alpha_{m} \beta_{m} \psi_{q}-\omega \beta_{m} \psi_{d}+u_{q} / \sigma_{m} ; \\
& \dot{\Psi}_{d}=-\alpha_{m} \psi_{d}+\alpha_{m} L_{m}\left(i_{m}\right) i_{d}+\omega_{2} \psi_{q} ; \\
& \dot{\Psi}_{q}=-\alpha_{m} \psi_{q}+\alpha_{m} L_{m}\left(i_{m}\right) i_{q}-\omega_{2} \psi_{d} ; \\
& \dot{\varepsilon}_{0}=\omega_{0},
\end{aligned}
$$


where $u_{d}, u_{q}$ are stator voltage components (here and throughout the paper subscripts $d$ and $q$ denote vector variable components in the dq reference frame), $i_{d}, i_{q}$ are stator currents, $\psi_{d}, \psi_{q}$ define the rotor flux, $\omega$ is the rotor speed, $T$ is the electromagnetic torque, $T_{L}$ is the load torque and $\varepsilon_{0}$ is the angular position of the dq reference frame with respect to a fixed stator reference frame (a-b) in which physical variables are defined. Slip frequency is defined as $\omega_{2}=\omega_{0}-\omega$, and $J$ is the total rotor inertia. One pole pair is assumed without loss of generality. In the model (1) constants (all positive) related to IM electrical parameters are given by:

$$
\begin{aligned}
& \alpha_{m}=R_{2} / L_{2}\left(i_{m}\right), \beta_{m}=L_{m}\left(i_{m}\right) /\left[L_{2}\left(i_{m}\right) \sigma_{m}\right], \\
& \gamma_{m}=R_{1} \sigma_{m}^{-1}+\alpha_{m} \beta_{m} L_{m}\left(i_{m}\right), \mu_{1 m}=3 L_{m}\left(i_{m}\right) /\left[2 L_{2}\left(i_{m}\right)\right], \\
& \sigma_{m}=L_{1}\left(i_{m}\right)-L_{m}^{2}\left(i_{m}\right) / L_{2}\left(i_{m}\right),
\end{aligned}
$$

Here $R_{1}, R_{2}$ are stator and rotor resistances respectively. The index $\mathrm{m}$ in (2) is used to denote the parameter's dependency on magnetizing current $i_{m}$. From the practical assumption that there is a constant relationship between any pair of $L_{1}\left(i_{m}\right), L_{2}\left(i_{m}\right)$ and $L_{m}\left(i_{m}\right)$ it can be concluded that: $\alpha_{m} L_{m}\left(i_{m}\right)=\alpha L_{m}=$ const , $\mu_{1 m}=\mu_{1}=$ const , where $L_{m}=$ const - the machine magnetizing inductance at the rated flux.

\section{CONTROL PROBLEM STATEMENT}

Consider the IM model (1) and assume that: the torque reference trajectory $T^{*}$ is a smooth and bounded function together with its first and second time derivatives; stator currents and rotor speed are available for measurement; all motor parameters are known and constant; all saturation-dependent parameters are known function of a magnetizing current.

Under these assumptions, the control problem is to design a torque controller which guarantees the following control objectives: CO1. Asymptotic torque tracking with all internal signals bounded, i.e. $\lim _{t \rightarrow \infty} \tilde{T}=0$, where $\tilde{T}=T-T^{*}$ is torque tracking error. CO2. Maximization of Torque per Ampere ratio in steady state: $\max \left(T / I_{1}\right), I_{1}=\sqrt{i_{d}^{2}+i_{q}^{2}}$ is a stator current magnitude. CO3. Asymptotic field orientation, i.e. $\lim _{t \rightarrow \infty} \psi_{q}=0$.

\section{CONTROL ALGORITHM DESIGN}

Following the design procedure [7] in this section a direct field-oriented torque-flux tracking control employing an asymptotic closed loop rotor flux observer is developed.

The reduced-order flux observer is defined as:

$$
\begin{aligned}
& \dot{\hat{\psi}}=-\alpha_{m} \hat{\psi}+\alpha L_{m} i_{d}, \\
& \dot{\varepsilon}_{0}=\omega_{0}=\omega+\alpha L_{m} i_{q} / \hat{\psi}+v_{\varepsilon} / \hat{\psi}, \\
& \dot{\hat{i}}_{d}=-\gamma_{m} \hat{i}_{d}+\omega_{0} i_{q}+\alpha_{m} \beta_{m} \hat{\psi}+\sigma_{m}^{-1} u_{d}+k_{1} \tilde{\tilde{i}}_{d},
\end{aligned}
$$

where $\hat{\psi}$ is an observed flux value, $\hat{i}_{d}$ - estimation of d-axis current, $\tilde{\tilde{i}}_{d}=i_{d}-\hat{i}_{d}-$ current estimation error, $\gamma_{1}>0$ is the observer correction gain and $v_{\varepsilon}$ is observer correction signal to be designed later.
Let define the flux estimation errors

$$
\tilde{\psi}_{d}=\psi_{d}-\hat{\psi}, \tilde{\psi}_{q}=\psi_{q} .
$$

From (1) and (3) the flux-current estimation error dynamic is defined by

$$
\begin{aligned}
& \dot{\tilde{\Psi}}_{d}=-\alpha_{m} \tilde{\Psi}_{d}+\omega_{2} \tilde{\Psi}_{q} ; \\
& \dot{\tilde{\Psi}}_{q}=-\alpha_{m} \tilde{\Psi}_{q}-\omega_{2} \tilde{\Psi}_{d}-v_{\varepsilon} ; \\
& \dot{\tilde{i}}_{d}=-k_{1} \tilde{\tilde{i}}_{d}+\alpha_{m} \beta_{m} \tilde{\Psi}_{d}+\beta_{m} \omega \tilde{\Psi}_{q} .
\end{aligned}
$$

Selecting $v_{\varepsilon}=\gamma_{1} \beta_{m} \omega \tilde{\tilde{i}}_{d}$ using Lyapunov stability criteria it can be concluded that the equilibrium point $\left(\tilde{\Psi}_{d}, \tilde{\Psi}_{q}, \tilde{\tilde{i}}_{d}\right)^{T}=0$ is globally exponentially stable if $k_{i d}>\gamma_{1} \alpha_{m} \beta_{m}^{2} / 4$.

A feedback-linearizing torque and flux controllers are constructed as [7]:

$$
\begin{gathered}
i_{q}=T^{*} / \mu_{1} \hat{\psi}, \\
i_{d}=\left(\alpha_{m} \psi^{*}+\dot{\psi}^{*}-k_{\psi p} \tilde{\psi}-x_{\psi}\right) / \alpha L_{m}, \dot{x}_{\psi}=k_{\psi i} \tilde{\psi},
\end{gathered}
$$

where: $\tilde{\psi}=\hat{\psi}-\psi^{*}$ is flux (estimated) tracking error; $k_{\psi p}$ and $k_{\psi i}$ are the controller proportional and integral gains respectively.

The stability analysis similar to given in [7] proofs that control objectives $(\mathrm{CO} 1),(\mathrm{CO} 3)$, flux reference $\left(\psi^{*}>0\right)$ tracking and flux vector estimation are globally achieved.

It is important to note that the correction term $v_{\varepsilon}=\gamma_{1} \beta_{m} \omega \tilde{\tilde{i}}_{d}$ provides the closed loop properties and therefore the observer is robust with respect to the rotor resistance variations at nonzero machine speed [8]. In contrast to solution [7] the proposed controller uses d-axis current estimation error instead of current tracking error for compensation term $\mathrm{v}_{\varepsilon}$. This allows to use the d-axis current PI controller and therefore improves the robustness with respect to stator resistance variations or inverter non-idealities.

The currents in (6), (7) represent their desired dynamics given by the trajectories of $i_{d}{ }^{*}$ and $i_{q}{ }^{*}$. Let us define current tracking errors $\tilde{i}_{d}=i_{d}-i_{d}^{*}, \quad \tilde{i}_{q}=i_{q}-i_{q}^{*}$. The current loops controls are constructed as follows:

$$
\begin{gathered}
u_{d}=\sigma_{m}\left(\gamma_{m} i_{d}^{*}-\omega_{0} i_{q}-\alpha_{m} \beta_{m} \hat{\psi}+\dot{i}_{d}^{*}-k_{i p d} \tilde{i}_{d}+x_{d}\right), \dot{x}_{d}=-k_{i i d} \tilde{i}_{d}, \\
u_{q}=\sigma_{m}\left(\gamma_{m} i_{q}^{*}+\omega_{0} i_{d}+\beta_{m} \omega \hat{\psi}+i_{q}^{*}-k_{i p q} \tilde{i}_{q}+x_{q}\right), \dot{x}_{q}=-k_{i i q} \tilde{i}_{q},
\end{gathered}
$$

where $k_{i p d}, k_{i p q}$ are the current controller's proportional gains, $k_{i i q}, k_{i i d}$ are the integral gains and $x_{d}, x_{q}$ are the integral components of the current controllers.

Using stability analysis similar to given in [8] we conclude that for a bounded speed signal $\omega$ the equilibrium point $\left(\tilde{\psi}_{d}, \tilde{\psi}_{q}, \tilde{\tilde{i}}_{d}\right)^{T}=0,\left(\tilde{i}_{d}, x_{q}, \tilde{i}_{q}, x_{q}\right)^{T}=0,\left(\tilde{\psi}, x_{\psi}\right)^{T}=0$ is globally exponentially stable. This implies that the torque tracking error $\tilde{T}\left(T^{*}, \tilde{i}_{d}, \tilde{i}_{q}, \tilde{\psi}_{d}, \tilde{\psi}_{q}\right)$ decays exponentially to zero, while also achieving asymptotic field orientation. Hence the control objectives (CO1) and (CO3) are met. 
The torque-flux tracking capabilities of the controller (3), (6), (7), (8) and (9) allow the selection of flux reference trajectories such that the maximization of Torque per Ampere ratio in steady state $(\mathrm{CO} 2)$ is achieved:

$$
\psi^{*}\left(T^{*}\right)=L_{m}\left(i_{d}\left(T^{*}\right)\right) i_{d}\left(T^{*}\right)
$$

Example curves for $i_{d}\left(T^{*}\right)$ and $\psi^{*}\left(T^{*}\right)$ are depicted in Fig. 1 for the IM with rated power of $5.5 \mathrm{~kW}$ (see Appendix).

The block diagram of the torque control system with the feedback linearizing controller (3), (6), (7), (8) and (9) for saturated IM is shown in Fig.2.
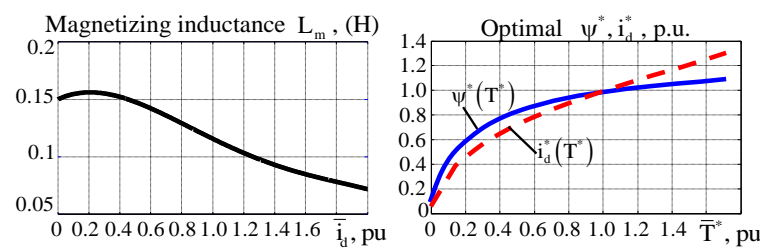

Fig. 1. MTPA relationships for the IM in Appendix I.

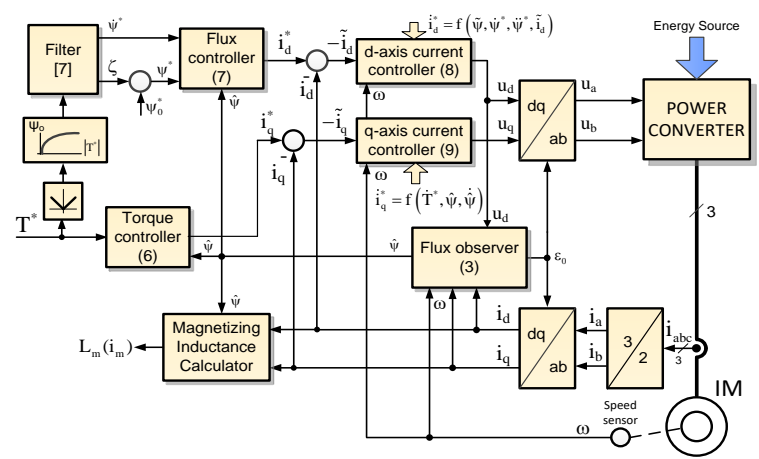

Fig. 2. Simplified block diagram of the torque control system.

\section{EXPERIMENTAL VERIFICATION}

This Section reports the results of experiments conducted to support the analytical findings of the previous Section. The test rig was a Rapid Prototyping Station (RPS) as shown in Fig. 3. The RPS was based on a $5.5 \mathrm{~kW}$ (see data in Appendix) induction motor (IM\#1 in Fig. 3), controlled by a 380V/50A PWM-inverter. The IM was mechanically coupled to a vectorcontrolled load machine. The motor speed and torque were measured by a 2500ppr optical encoder and a torque sensor (Lorenz Messtechnik DR-2). The sampling time was $100 \mu \mathrm{s}$.

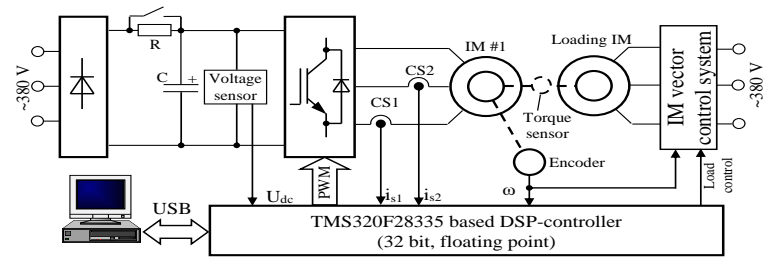

Fig. 3. Experimental setup.

It should be noted that during all experiments the machine rotor speed, when processing of the torque tracking reference, has been stabilized at $11 \mathrm{rad} / \mathrm{s}$ by the speed controlled load machine. Under these conditions, the mechanical power for rated torque is equal to $385 \mathrm{~W}$. Such test conditions are selected in order to demonstrate the effect of active losses optimization, which are not dependent from the rotor speed.

\section{A. Comparison of torque tracking performances}

The task of this experiment was to assess the effect of magnetic saturation on torque tracking performance and MTPA ratio for three different control strategies, namely: Indirect Field-Oriented Control with constant flux (IFOC) [8]; MTPA control (3), (6), (7), (8) and (9) with linear magnetic curve representation (MTPA-L); MTPA control (3), (6), (7), (8) and (9) accounting for IM saturation (MTPA-S).

The core idea of this experiment was to demonstrate both the error in torque regulation under MTPA control, if the saturation is not accounted for (MTPA-L), and the capability of the MTPA-S algorithm to achieve an error margin which is compatible with IFOC (however IFOC does not provide MTPA criteria).

During the tests the following test scenario was applied: initial time interval $(t<0.5 \mathrm{~s})$ for setting flux reference for MTPA controllers $\left(\psi_{0}{ }^{*}=0.05 \mathrm{~Wb}\right)$ and for setting a rated excitation $\left(\psi^{*}=0.96 \mathrm{~Wb}\right)$ for IFOC strategy; starting at $t=0.5 \mathrm{~s}$, an incremental series of torque reference steps was applied as shown in Fig.4(a); the increments are $7 \mathrm{Nm}$ each with the duration $1.45 \mathrm{~s}$ such that at $t=6.55 \mathrm{~s}$ the torque reference reaches the machine rated value of $35 \mathrm{Nm}$; at $t=7.75 \mathrm{~s}$ the torque reference is reduced to zero. The results are presented in Fig. 4. It can be concluded from Fig. 4(b), that the IFOC algorithm provides asymptotic torque tracking.
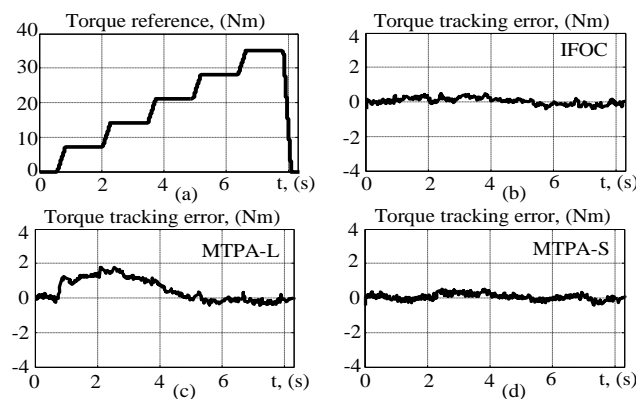

Fig. 4. Torque tracking: (a) torque reference trajectory; (b) $-(d)$ torque errors provided by IFOC (b), MTPA-L (c) and MTPA-S (d) controlers.

Under MTPA-L control (Fig. 4(c)) asymptotic torque tracking is only achieved when the rotor flux is close to its rated value. As the flux reduces, the flux estimation error increases (due to saturation) leading to a torque tracking error. For torque reference $7 \mathrm{Nm}$ the error is approximately $15 \%$. The MTPA-S control takes into account the effect of magnetic saturation; therefore, the torque tracking error is nearly zero in the full range of torque references, as the results in Fig.4(d) confirm. Hence, the proposed MTPA-S controller provides the same accuracy as the IFOC in the whole torque regulation range. From the experimental results in Fig.4 it is clear that the proposed MTPA-S strategy successfully compensates for the IM saturation effect.

\section{B. Comparison of torque per Ampere ratio}

During the second test, modified operation sequence was applied (Fig. 5): initial time interval $(\mathrm{t}<1 \mathrm{~s})$ for establishing the minimum flux $\psi_{0}=0.02 \mathrm{~Wb}$; at $t=1 \mathrm{~s}$ a $7 \mathrm{Nm}$ torque reference step (13\% of the rated torque) is applied followed by multiple $7 \mathrm{Nm}$ steps, each $1 \mathrm{~s}$ apart, until $35 \mathrm{Nm}$ is reached (100\% of rated value) at $t=5 \mathrm{~s}$, as illustrated by Fig. 4 ; at $t=6 \mathrm{~s}$ the torque reference is 
reduced to zero; during the interval $8 \mathrm{~s}$ to $15 \mathrm{~s}$ the torque reference is a sinusoidal function with magnitude $14 \mathrm{Nm}$ and angular frequency $2.25 \mathrm{rad} / \mathrm{s}$. This trajectory can be regarded as slow in comparison to IM magnetic system dynamics.

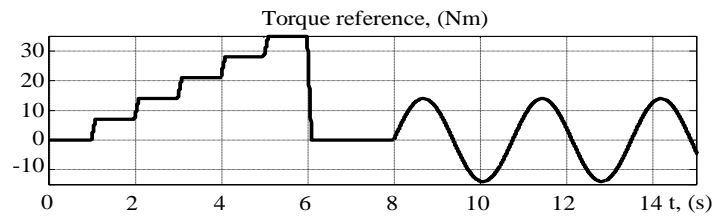

Fig. 5. Torque reference.
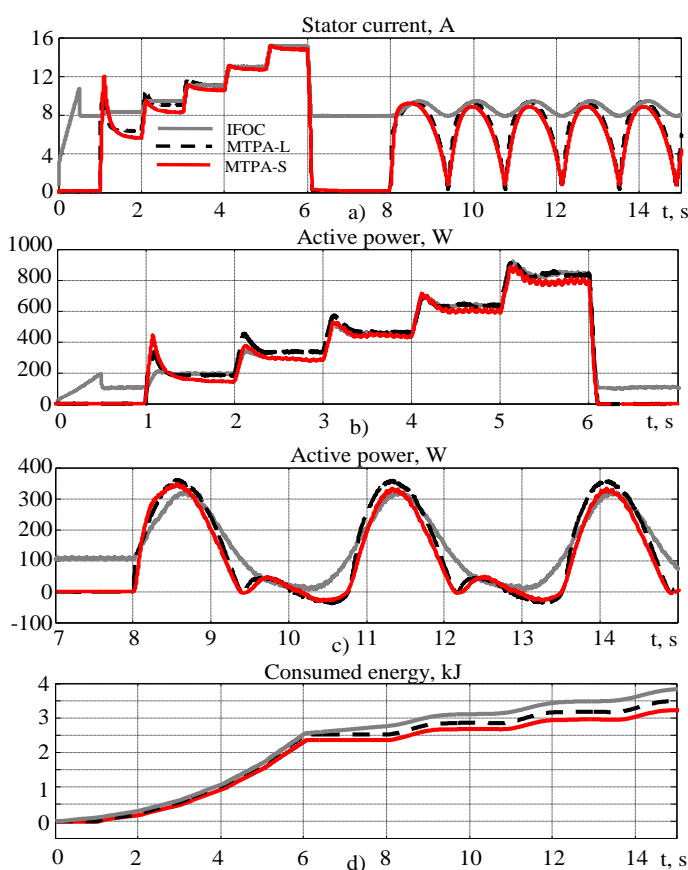

Fig. 6. Torque tracking: (a) stator current; (b)-(c) active power; (d) energy consumed during the test by IFOC, MTPA-L and MTPA-S controlers.

The stepped torque reference is used in order to show where the MTPA optimization can be achieved. The sinusoidal part of reference trajectory is used to demonstrate the system performance during torque zero crossing.

The stator current magnitude, machine active power and cumulative consumed energy for compared control strategies are shown in Fig. 6. As it follows from Fig. 6(a) IFOC requires the constant current at the level of 40-50 \% from the rated value in order to produce the constant flux even at zero torque. MTPA-L control provides MTPA optimization only for limited torque values. From the current transients in Fig. 6(a) it can be concluded, that for the motor under test the stator current behavior for IFOC and MTPA- L becomes the same beyond the torque reference of $14 \mathrm{Nm}$ (40\% from rated torque).

At the same time MTPA-S control takes into account the machine magnetizing curve for each torque reference point. Due to this, the MTPA optimization is achieved for all torque references and the steady state current values are less in comparison with two other controllers resulting in higher torque per Ampere ratio.

The results in Fig. 6 (b) - (d) clearly demonstrate the advantages of MTPA-S control. It can be seen that MTPA-S controller requires the smaller values of active power for the most parts of torque reference. As the result the total consumed energy during the test for MTPA-S controller is equal to $3240 \mathrm{~J}$ and for MTPA-L one - $3500 \mathrm{~J}$. In comparison with IFOC $(3850 \mathrm{~J})$ the improvement of $16 \%$ and $9 \%$ is achieved. The efficiency at rated torque under the test conditions is $45.6 \%$ for IFOC and MTPA-L control and $48.4 \%$ for MTPA-S control.

\section{CONCLUSIONS}

In this paper an improved MTPA field-oriented control algorithm for induction motor is presented. The controller guarantees asymptotic torque tracking of smooth reference trajectories and maximises the torque per Ampere ratio when the machine is operating with constant or slow varying torque. In contrast to existing author's solution, proposed controller uses daxis current estimation error instead of current tracking error for flux subsystem robustification. This allows to use the d-axis current PI controller and therefore improves the robustness with respect to stator resistance variations or inverter non-idealities.

An intensive experimental study of the proposed solution confirms that in comparison with traditional field-oriented controller with constant flux, for the same torque transient performances a significant stator current reduction is achieved in steady-state operation. Improved efficiency and the effectiveness of the magnetic system saturation compensation are also demonstrated experimentally.

\section{APPENDIX}

IM \#1 technical characteristics: $P_{n}=5.5 \mathrm{~kW}, I_{n}=11 \mathrm{~A}$, $V_{n}=380 \mathrm{~V}, f_{n}=50 \mathrm{~Hz}, \omega_{n}=150 \mathrm{rad} / \mathrm{s}, \quad R_{1}=0.94 \Omega, R_{2}=0.65 \Omega$, $L_{1 \sigma}=L_{2 \sigma}=0.006 \mathrm{H}, L_{m}=0.117 \mathrm{H}$.

Controller parameters: $\quad k_{i p d}=k_{i p q}=700, \quad k_{i i d}=k_{i i q}=122500$, $\gamma_{1}=8 \cdot 10^{-3}, k_{\psi \mathrm{p}}=30, k_{\psi \mathrm{i}}=450$.

\section{REFERENCES}

[1] B.K.Bose Modern power electronics and AC Drives, Upper Saddle River, NJ: Prentice Hall, 2002.

[2] A. M. Bazzi and P. T. Krein, "Review of methods for real-time loss minimization in induction machines," IEEE Trans. on Industry Applications, vol. 46, no. 6, pp. 2319-2328, 2010.

[3] S. A. Odhano, R. Bojoi, A. Boglietti, Ş. G. Roşu, G. Griva "Maximum efficiency per torque direct flux vector control of induction motor drives" IEEE Trans. Industry Applications, vol. 51, no. 6, pp. 4415 - 4424, 2015.

[4] O.Wasynchuk, S.D.Sudhoff, K.A.Corsine, J.Tichenor, P.Krause, I.Hansen, L. Taylor "A maximum torque per Ampere control strategy for induction motor drives" IEEE Trans. on Energy Conversion, vol.13, no.2, pp.163169, 1998.

[5] C.Kwon and S.D.Sudhoff, "An improved maximum torque per amp control strategy for induction machine drives" in 20th Annual IEEE Applied Power Electronics Conference and Exposition, March, 2005, pp. 740-745.

[6] R.Bojoi, Z.Li, S.A.Odhano, G.Griva, A.Tenconi "Unified direct-flux vector control of induction motor drives with maximum torque per ampere operation" in Proc. 2013 Energy Conversion Congress and Exposition, 1519 Sep., Denver, 2013, pp.3888-3895.

[7] S. Bozhko, S. Dymko, S. Kovbasa and S. M. Peresada, "Maximum Torqueper-Amp Control for Traction IM Drives: Theory and Experimental Results," in IEEE Trans. on Industry Applications, vol. 53, no. 1, pp. 181193, Jan.-Feb. 2017.

[8] S.Peresada and A.Tonielli, "High-performance robust speed-flux tracking controller for induction motor,” Int. J. Adapt. Control Signal Process., vol. 14, pp. 177-200, 2000. 